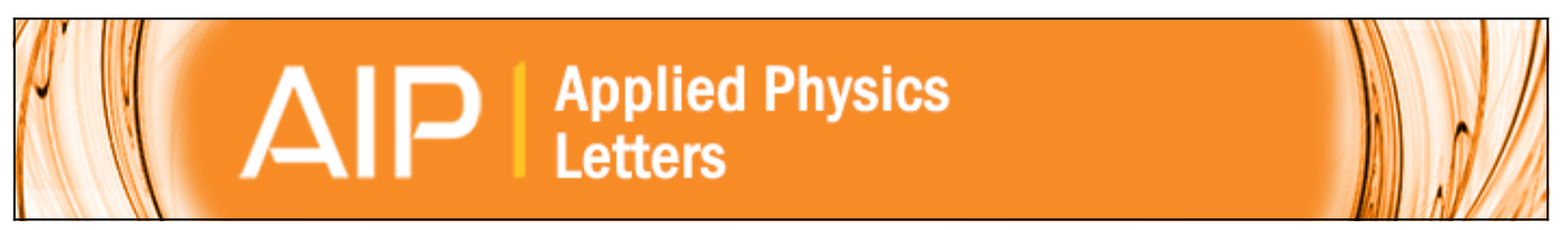

\title{
A-centers in silicon studied with hybrid density functional theory
}

H. Wang, A. Chroneos, C. A. Londos, E. N. Sgourou, and U. Schwingenschlögl

Citation: Applied Physics Letters 103, 052101 (2013); doi: 10.1063/1.4817012

View online: http://dx.doi.org/10.1063/1.4817012

View Table of Contents: http://scitation.aip.org/content/aip/journal/apl/103/5?ver=pdfcov

Published by the AIP Publishing 


\title{
A-centers in silicon studied with hybrid density functional theory
}

\author{
H. Wang, ${ }^{1}$ A. Chroneos, ${ }^{2,3, a)}$ C. A. Londos, ${ }^{4}$ E. N. Sgourou, ${ }^{4}$ and U. Schwingenschlögl ${ }^{1, b)}$ \\ ${ }^{1}$ PSE Division, KAUST, Thuwal 23955-6900, Saudi Arabia \\ ${ }^{2}$ Materials Engineering, The Open University, Milton Keynes MK7 6AA, United Kingdom \\ ${ }^{3}$ Department of Materials, Imperial College, London SW7 2AZ, United Kingdom \\ ${ }^{4}$ University of Athens, Solid State Physics Section, Panepistimiopolis Zografos, Athens 157 84, Greece
}

(Received 27 May 2013; accepted 15 July 2013; published online 29 July 2013)

\begin{abstract}
Density functional theory employing hybrid functional is used to gain fundamental insight into the interaction of vacancies with oxygen interstitials to form defects known as A-centers in silicon. We calculate the formation energy of the defect with respect to the Fermi energy for all possible charge states. It is found that the neutral and doubly negatively charged A-centers dominate. The findings are analyzed in terms of the density of states and discussed in view of previous experimental and theoretical studies. (C) 2013 AIP Publishing LLC. [http://dx.doi.org/10.1063/1.4817012]
\end{abstract}

Silicon ( $\mathrm{Si}$ ) is widely used in a range of microelectronic, photovoltaic, and sensor devices. However, detailed understanding of many defect-dopant interactions that can affect its properties is not well established. ${ }^{1-4}$ Such dopant-defect interactions become increasingly important as the characteristic dimensions of devices nowadays are a few nanometers. Oxygen $(\mathrm{O})$ is a significant impurity in Czochralski-Si and can be introduced in high concentrations during crystal growth. The oxygen intersitials $\left(\mathrm{O}_{\mathrm{i}}\right)$ can readily bind to vacancies $(V)$ forming $V O$ pairs (via $V+\mathrm{O}_{\mathrm{i}} \rightarrow V \mathrm{O}$ ). ${ }^{5,6}$ As such A-centers are both electrically and optically active, it is technologically important to suppress their formation. For this reason various defect engineering strategies have been proposed. ${ }^{7,8}$ In particular, A-centers can have a deleterious impact on Si-based imaging and spectroscopy sensors (complementary metal-oxide-semiconductor sensors and charge-coupled device sensors) in space. The formation of A-centers is enhanced in this radiation environment as highenergy particles (for example, protons and neutrons) cause lattice displacement damage giving rise to a supersaturation of vacancies. These vacancies diffuse through the lattice binding with $\mathrm{O}_{\mathrm{i}}$ to form A-centers, which are effective traps that negatively impact the device performance. In the present study, we use hybrid density functional theory to investigate the ionization and formation energies of the $V O$ defect with respect to the Fermi energy for all reasonable charge states. For the dominant charge states, we address the density of states (DOS).

The Vienna Ab-initio Simulation Package ${ }^{9}$ is used with pseudopotentials being generated by the projector augmented wave method. ${ }^{10}$ Also, the PBEsol ${ }^{11}$ functional is employed. Employing a $2 \times 2 \times 2$ supercell (containing $64 \mathrm{Si}$ atoms), we have tested the convergence of the k-point mesh within the Monkhorst-Pack scheme ${ }^{12}$ from $2 \times 2 \times 2$ to $6 \times 6 \times 6$ meshes with the cutoff energy for the plane waves set to $400 \mathrm{eV}$. The total energy difference between the $3 \times 3 \times 3$ and $6 \times 6 \times 6$ calculations is found to be less than $0.13 \mathrm{meV}$ per atom. Afterwards the k-point mesh has been kept as $4 \times 4 \times 4$ and the cutoff energy increased from 300 to $500 \mathrm{eV}$

\footnotetext{
${ }^{\text {a)} A l e x . C h r o n e o s @ o p e n . a c . u k ~}$

${ }^{\text {b)} U d o . S c h w i n g e n s c h l o g l @ k a u s t . e d u . s a ~}$
}

with a step of $50 \mathrm{eV}$. The difference between the results of the 400 and $500 \mathrm{eV}$ calculations turned out to be less than $0.1 \mathrm{meV}$ per atom. Considering that hybrid functional calculations are extremely time consuming, we have decided to choose the $3 \times 3 \times 3$ mesh and $400 \mathrm{eV}$ cutoff energy, which can guarantee a good accuracy, for all following calculations. Then the formation energy of a $V$ has been calculated for a larger $2 \times 2 \times 4$ supercell (containing $128 \mathrm{Si}$ atoms) to be $3.52 \mathrm{eV}$, while it is $3.56 \mathrm{eV}$ for the $2 \times 2 \times 2$ supercell. The difference is small so that the $2 \times 2 \times 2$ supercell is found to be large enough. The lattice constant of pure $\mathrm{Si}(2 \times 2 \times 2$ supercell) optimized by the PBEsol functional is very close to the one obtained by hybrid functional Heyd, Scuseria, and Ernzerhof (HSE) calculations. ${ }^{13}$ For each defect structure (with or without charge), the lattice constant is set to be the same as that of pure $\mathrm{Si}$, with the atomic positions being relaxed for the forces on all atoms to decline below $0.01 \mathrm{eV} /$ $\AA$. The optimized structures are used for the HSE calculations ${ }^{14-16}$ with a screening parameter of $\mu=2.06 \AA^{-1}$. The local contribution is calculated by the PBE functional. ${ }^{17}$ To eliminate the artificial interaction between the charged defects introduced by periodic boundary conditions, we employ the correction approach developed by Freysoldt and coworkers ${ }^{18,19}$ to our data. The dielectric constant used in this approach is taken from Ref. 20.

Formation energies of the neutral and charged $V O$ defects in Si are calculated using the relation ${ }^{21}$

$$
\Delta \mathrm{H}_{D, q}\left(\mu_{e}, \mu_{\alpha}\right)=E_{D, q}-E_{H}+\sum_{\alpha} n_{\alpha} \mu_{\alpha}+q \mu_{e}
$$

where $E_{D, q}$ is the total energy of the defective cell with a charge $q$ and $E_{H}$ is the total energy of the perfect Si cell. Moreover, $n_{\alpha}$ is the number of atoms added to or removed from the defective cell and $\mu_{\alpha}$ corresponds to the chemical potentials. Finally, $\mu_{e}$ is the Fermi energy measured from the top of the valence band maximum (VBM), with values lying in the band gap: $E_{V B M} \leq \mu_{e} \leq E_{V B M}+E_{g}$. The chemical potential of $\mathrm{O}$ is calculated using quartz $\mathrm{SiO}_{2}$.

In previous experimental studies, it was determined that the A-center can exist at different charge states depending on the position of the Fermi level in the band gap. ${ }^{22,23}$ Figure 1 


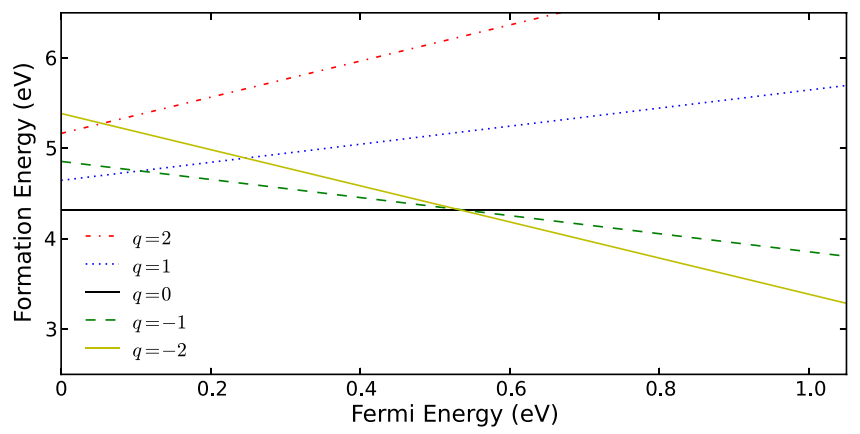

FIG. 1. Formation energies of the $V O$ defect, with respect to the Fermi energy.

presents the formation energies of the $V O$ defect with respect to the Fermi energy for various charge states. The ionization energies for the defects (derived from Figs. 1 and 2 as the Fermi energy point where the respective formation energies cross) are summarized in Table I. From Fig. 1, it is deduced that the $V \mathrm{O}^{0}$ defect dominates up to a Fermi energy of $0.54 \mathrm{eV}$, above which the $V \mathrm{O}^{-2}$ defect becomes dominant. Density functional theory strictly applies to a temperature of $0 \mathrm{~K}$, whereas in experimental studies the temperature can impact the results. For example, it was determined by Bean and Newman ${ }^{23}$ that an increase of the temperature will effectively lower the position of the Fermi level in the band gap and in turn reduce the proportion of the $V \mathrm{O}^{-1}$ with respect to the $V \mathrm{O}^{0}$ defect. In most previous studies based on density functional theory, the investigation of the $V \mathrm{O}^{-2}$ defect was omitted but the $V \mathrm{O}^{0}$ and $V \mathrm{O}^{-1}$ defects were analyzed in detail. ${ }^{24}$ Interestingly, Pesola and coworkers ${ }^{25}$ employing density functional theory within the local density approximation, have considered the doubly negatively charged state of the A-center and calculated that it is prevalent for Fermi energies above $0.53 \mathrm{eV}$ in agreement with the present study (note the value $0.53 \mathrm{eV}$ for configuration $(-,--)$ in Table I). They have also calculated that $V \mathrm{O}^{-1}$ is prevalent in the Fermi energy range $0.4-0.53 \mathrm{eV}$, which, on the other hand, is not in agreement with the present study. One should notice that the structures have been optimized by the PBEsol functional, which gives accurate results for $\mathrm{Si}^{13,26}$ In our present study, we obtain a lattice constant of pure $\mathrm{Si}$ that is only $0.1 \%$ larger than the experimental value. ${ }^{27}$ In addition, it is expected that the HSE functional constitutes the most appropriate theoretical approach to the electronic states, because it reproduces the experimental band gap of $\mathrm{Si}^{13,20,28}$

TABLE I. Calculated ionization energies (in $\mathrm{eV}$ ) for the $V \mathrm{O}$ and $V$ defects.

\begin{tabular}{lcc}
\hline \hline & $V \mathrm{O}$ & $V$ \\
\hline$(++/ 0)$ & $\ldots$ & 0.04 \\
$(+/ 0)$ & $\ldots$ & $\ldots$ \\
$(0 /-)$ & 0.54 & 0.33 \\
$(0 /--)$ & 0.54 & 0.27 \\
$(-/--)$ & 0.53 & 0.21 \\
$(+/-)$ & 0.11 & 0.17 \\
$(+/--)$ & 0.25 & 0.18 \\
$(++/-)$ & $\ldots$ & 0.14 \\
$(++/--)$ & 0.05 & 0.16 \\
\hline \hline
\end{tabular}
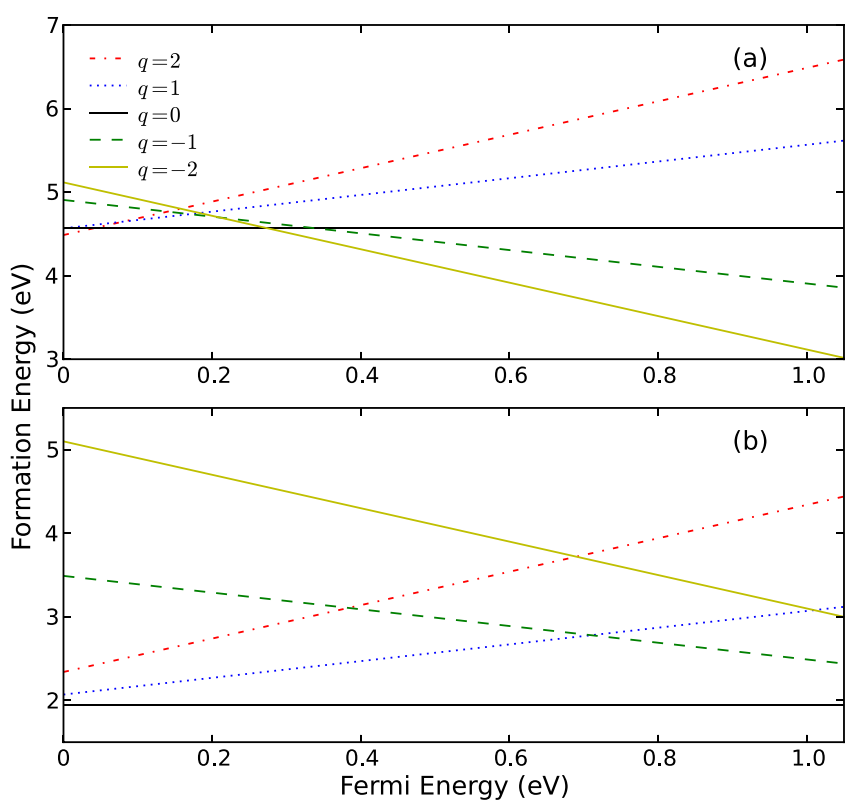

FIG. 2. Formation energies of the (a) $V$ and (b) $\mathrm{O}_{\mathrm{i}}$ defects, with respect to the Fermi energy.

To gain further understanding on the formation of the $V O$ defect, we consider next the formation energies of the $V$ and $\mathrm{O}_{\mathrm{i}}$ defects (i.e., the constituents of the $V \mathrm{O}$ defect), with respect to the Fermi energy for various charge states. Figure 2(a) illustrates that the formation energy of $V$ is around $4.5 \mathrm{eV}$ (which agrees with the theoretical result of $4.1 \mathrm{eV}$ obtained by Blöchl et $a l .^{29}$ ) at low Fermi energy and decreases to about $3 \mathrm{eV}$ for the -2 charge state, which is favourable when the Fermi energy is above $0.27 \mathrm{eV}$ (Table I). Consequently, $V$ is difficult to form in the whole Fermi energy range. The high formation energy of $V \mathrm{~s}$ in $\mathrm{Si}$ is consistent with comprehensive analyses of Si crystal growth, high temperature wafer processing, $\mathrm{Si}$ self-diffusion, and metal/dopant diffusion experiments. ${ }^{30-32}$ For example, the previous study of Voronkov and Falster ${ }^{31}$ has determined a vacancy formation energy of $3.95 \mathrm{eV}$ in agreement with our findings $(4.12 \mathrm{eV}$ at midgap). Earlier experiments have claimed a value of $3.6 \pm 0.2 \mathrm{eV} .{ }^{30}$ Nevertheless, in a radiation environment there is a supersaturation of vacancies, which will benefit the formation of A-centers. As it can be seen from Figure 2(b), the neutral charge state is dominant for the $\mathrm{O}_{\mathrm{i}}$ defect with a formation energy of $1.95 \mathrm{eV}$ which agrees with the result in Ref. 25. In a simplistic approach, one may consider that the formation of the $\mathrm{VO}^{0}$ and $\mathrm{VO}^{-2}$ defects occurs via the $V^{0}+\mathrm{O}_{\mathrm{i}} \rightarrow V \mathrm{O}$ and $V^{-2}+\mathrm{O}_{\mathrm{i}} \rightarrow V \mathrm{O}^{-2}$ reactions, respectively.

In Fig. 3, comparison of the shaded and unshaded total DOSs indicates that the band gap of pure Si obtained by the HSE scheme is about $1.05 \mathrm{eV}$. The calculated band gap, thus, is in excellent agreement with the experimental value of $1.17 \mathrm{eV} .{ }^{26}$ We present the total DOSs of the $V O$ defect in the charge states $0,-1$, and -2 , since these are most important states according to Fig 1. The valence and conduction bands appear almost at the same energy for the different charge states, where the valence bands shift a bit down in energy as compared to pure $\mathrm{Si}$. The $V \mathrm{O}$ defect introduces 


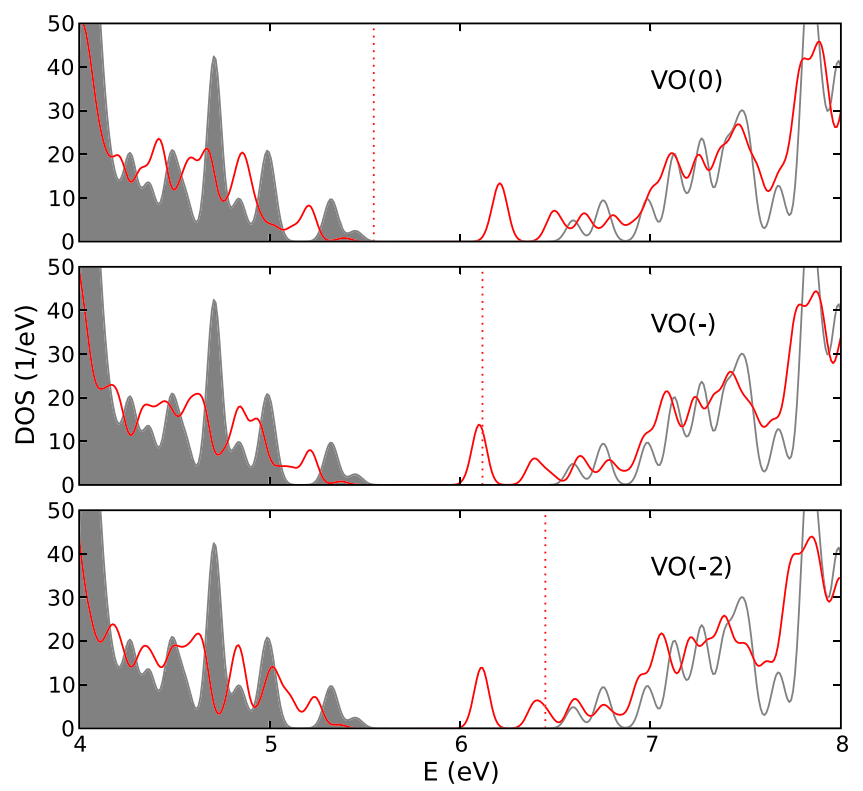

FIG. 3. Total DOSs of pure Si (gray) and Si with $V O$ defect (red). The occupied states of pure $\mathrm{Si}$ are shaded. For the $V \mathrm{O}$ defect, the states below the dotted line are occupied.

two holes in the electronic structure. For charge neutral VO, the empty defect states emerge about $0.5 \mathrm{eV}$ above the valence bands. Notably, the defect states consist not only of the peak at $6.1 \mathrm{eV}$ but also include a small portion which merges to the conduction bands. There is a small energy gap between the peak and that portion. This scenario is clarified by the DOSs of $V O$ in the -1 and -2 charge states. The peak of the defect states is occupied more than half in the DOS of the -1 charge state and a small part of the conduction bands is occupied further for the -2 charge state. Although there appears no distinct peak at the Fermi level in the DOS of the -1 charge state, we have also performed spin polarized calculation within the PBEsol functional scheme. The total energy is lowered by only $0.003 \mathrm{eV}$ as compared to the spin degenerate calculation, while we observe a total magnetic moment of $0.12 \mu_{\mathrm{B}}$. This indicates that inclusion of the spin polarization in the calculation is not important in the present case. The spin degenerate result is rather fully valid, demonstrating that the -1 charge state is not as stable as the -2 charge state, in agreement with the result that the -2 charge state dominates in the high Fermi energy range in Fig. 1.

Although our calculations generally do not favor the $V \mathrm{O}^{-1}$ state, they show agreement with other experimental quantities. First, the total DOS of $V \mathrm{O}^{-1}$ in Fig. 3 indicates that the Fermi level is about $0.16 \mathrm{eV}$ below the conduction band minimum, which is almost the value $(0.17 \mathrm{eV})$ measured by Watkins et al. ${ }^{33}$ Second, spin polarized PBEsol results for $\mathrm{VO}^{-1}$ show that the magnetic moment on the two $\mathrm{Si}$ atoms adjacent to the vacancy amounts to $56 \%$ of the total magnetic moment. This value is close to the $70 \%$ derived from the hyperfine interaction constant of the nonequivalent sites around the A-center. ${ }^{22}$

The ionization and formation energies as well as DOSs of A-centers in silicon have been investigated using hybrid density functional theory. The $V \mathrm{O}^{0}$ defect is found to dominate up to Fermi energy of $0.54 \mathrm{eV}$ and the $V \mathrm{O}^{-2}$ defect for higher Fermi energy. DOSs obtained for the different charge states of the $V O$ defect turn out to be in line with this picture, confirming prevalence of the $\mathrm{VO}^{0}$ and $V \mathrm{O}^{-2}$ states. Our results demonstrate that the specific methodology applied in first principles calculations plays an outstanding role. As compared to standard density functional theory, state-of-theart hybrid functional calculations for Si based systems result in band gaps very close to experimental values and therefore can be expected to give much improved descriptions of defects, including A-centers. Interestingly, the present theoretical results reveal the possibility of double negative charge A-centers in Si, whereas early experiments assign the single negative charge state at $n$-type doping conditions. This difference may be due to the different conditions of experiment (radiation damage, other defects) and theory (perfect lattice with a single A-center at $0 \mathrm{~K}$ ). At any rate, the possibility of determining $\mathrm{VO}^{-2}$ defects reconciling the two approaches should be investigated further. In that respect, an extension of low temperature experiments with O-rich Czochralski-Si for a range of doping ( $p$-type and $n$-type) and high irradiation dose could provide additional information about the negative charge states of the $V O$ defect. In addition, these measurements should be coupled with infrared spectroscopy measurements performed at various temperatures so that the shift of the Fermi level with temperature will help the defect to convert from one charge state to another thus enabling the possible identification of the $\mathrm{VO}^{-2}$ defects.

The authors thank Dr. David Hall (The Open University) for valuable discussions.

${ }^{1}$ D. Caliste and P. Pochet, Phys. Rev. Lett. 97, 135901 (2006).

${ }^{2}$ D. Caliste, P. Pochet, T. Deutsch, and F. Lançon, Phys. Rev. B 75, 125203 (2007).

${ }^{3}$ A. Chroneos, C. Jiang, R. W. Grimes, U. Schwingenschlögl, and H. Bracht, Appl. Phys. Lett. 94, 252104 (2009); A. Chroneos and C. A. Londos, J. Appl. Phys. 107, 093518 (2010).

${ }^{4}$ C. Gao, X. Ma, J. Zhao, and D. Yang, J. Appl. Phys. 113, 093511 (2013).

${ }^{5}$ R. C. Newman and R. Jones, "Oxygen in silicon" in Semiconductors and Semimetals, edited by F. Shimura (Academic Press, Orlando, 1994), Vol. 42, p. 289; C. A. Londos, Phys. Status Solidi A 113, 503 (1989).

${ }^{6}$ G. Davies and R. C. Newman, in Handbook of Semiconductors, edited by S. Mahajan (Elsevier, Amsterdam, 1994), Vol. 3, p. 1557.

${ }^{7}$ J. W. Corbett, G. D. Watkins, and R. S. McDonald, Phys. Rev. 135, A1381 (1964)

${ }^{8}$ A. Chroneos, C. A. Londos, E. N. Sgourou, and P. Pochet, Appl. Phys. Lett. 99, 241901 (2011)

${ }^{9}$ G. Kresse and D. Joubert, Phys. Rev. B 59, 1758 (1999).

${ }^{10}$ P. E. Blöchl, Phys. Rev. B 50, 17953 (1994).

${ }^{11}$ J. P. Perdew, A. Ruzsinszky, G. I. Csonka, O. A. Vydrov, G. E. Scuseria, L. A. Constantin, X. Zhou, and K. Burke, Phys. Rev. Lett. 100, 136406 (2008).

${ }^{12}$ H. J. Monkhorst and J. D. Pack, Phys. Rev. B 13, 5188 (1976).

${ }^{13}$ L. Schimka, J. Harl, and G. Kresse, J. Chem. Phys. 134, 024116 (2011).

${ }^{14}$ J. Heyd, G. E. Scuseria, and M. Ernzerhof, J. Chem. Phys. 118, 8207 (2003).

${ }^{15}$ J. Heyd and G. E. Scuseria, J. Chem. Phys. 121, 1187 (2004).

${ }^{16}$ J. Heyd, G. E. Scuseria, and M. Ernzerhof, J. Chem. Phys. 124, 219906 (2006).

${ }^{17}$ J. P. Perdew, M. Ernzerhof, and K. Burke, J. Chem. Phys. 105, 9982 (1996).

${ }^{18}$ C. Freysoldt, J. Neugebauer, and C. G. Van de Walle, Phys. Rev. Lett. 102, 016402 (2009).

${ }^{19}$ C. Freysoldt, J. Neugebauer, and C. G. Van de Walle, Phys. Status Solidi B 248, 1067 (2011).

${ }^{20}$ J. Paier, M. Marsman, and G. Kresse, Phys. Rev. B 78, 121201(R) (2008). 
${ }^{21}$ S. Lany and A. Zunger, Phys. Rev. B 78, 235104 (2008).

${ }^{22}$ G. D. Watkins and J. W. Corbett, Phys. Rev. 121, 1001 (1961).

${ }^{23}$ A. R. Bean and R. C. Newman, Solid State Commun. 9, 271 (1971).

${ }^{24}$ J. Coutinho, R. Jones, P. R. Briddon, and S. Öberg, Phys. Rev. B 62, 10824 (2000).

${ }^{25}$ M. Pesola, J. Von Boehm, T. Mattila, and R. M. Nieminen, Phys. Rev. B 60, 11449 (1999).

${ }^{26}$ P. Haas, F. Tran, and P. Blaha, Phys. Rev. B 79, 085104 (2009).

${ }^{27}$ R. W. G. Wyckoff, Crystal Structures, 2nd ed. (Interscience Publishers, New York, 1963), Vol. 1, p. 7-83.
${ }^{28}$ J. Heyd, J. E. Peralta, G. E. Scuseria, and R. L. Martin, J. Chem. Phys. 123, 174101 (2005).

${ }^{29}$ P. E. Blöchl, E. Smargiassi, R. Car, D. B. Laks, W. Andreoni, and S. T. Pantelides, Phys. Rev. Lett. 70, 2435 (1993).

${ }^{30}$ S. Dannefaer, P. Mascher, and D. Kerr, Phys. Rev. Lett. 56, 2195 (1986).

${ }^{31}$ V. V. Voronkov and R. Falster, Mater. Sci. Eng. B 134, 227 (2006).

${ }^{32}$ H. Bracht and A. Chroneos, J. Appl. Phys. 104, 076108 (2008).

${ }^{33}$ G. D. Watkins, J. W. Corbett, and R. M. Walker, J. Appl. Phys. 30, 1198 (1959). 